\title{
STOCHASTIC INTEGER WASTE MANAGEMENT PROBLEM SOLVED BY A MODIFIED PROGRESSIVE HEDGING ALGORITHM
}

\author{
Martin Málek ${ }^{1}$, Radovan Šomplák ${ }^{2}$, Pavel Popela ${ }^{1}$, and Jakub Kůdela ${ }^{3}$ \\ Brno University of Technology, Faculty of Mechanical Engineering \\ ${ }^{1}$ Institute of Mathematics \\ ${ }^{2}$ Institute of Process Engineering \\ ${ }^{3}$ Institute of Automation and Computer Science \\ Czech Republic \\ Jakub.Kudela@fme.vutbr.cz
}

\begin{abstract}
In this paper we describe a real-world large-scale stochastic integer waste-management decision making problem. The problem consists of choosing the optimal locations and capacities of new incineration plants, that will be used for the disposal of waste. To solve this problem, we implement a modified version of the progressive hedging algorithm. The presented case study with real-world data concerns the situation in the Czech Republic.
\end{abstract}

Keywords: waste management decision making, stochastic programming, facility location problem, progressive hedging algorithm

\section{Introduction}

Cautious waste management is one of the pivotal segments of the global environmental policies. As such, it possesses a multitude of opportunities for the operations research approaches (see [18]) to guide the decision making and to assess the possible outcomes and risks that result from the implementation of these decisions. Forecasting of the waste management related uncertain parameters (mainly the production of different kind of waste) was investigated in [17] and [19].

In this paper, our main concerns are the transportation and treatment of waste, and the decision on building new incinerator plants. The authors of [14] and [16] described the problem of designing test networks in waste management, while in [8] we can find a method for choosing the optimal capacity of a new plant. The transportation and facility location problems in waste management in an uncertain setting were investigated by [5] and [12]. A game-theoretic insight into the waste management problems can be found in [15].

In this paper, we describe a novel utilization of the Progressive Hedging algorithm [4] for the stochastic integer waste management problem as an alternative to the hybrid/metaheuristic approaches $[3,6,9]$ and to the Benders Decomposition method [10, 11].

\section{Model Building}

As was stated in the Introduction, our model will describe the waste management decision making problem of deciding on the location and capacity of new incinerator plants, that will be used for the treatment of waste. The objective encompasses the construction costs of the incinerators, the transportation costs on a road network, penalties for waste that is not properly disposed of (either in an incinerator or in a landfill) and the profits from waste treatment from the incinerator plants and landfills.

In the absence of uncertainty, the model would be a mixed-integer optimization problem (see [1]), but this is not our case. Since we do not know the amount of waste produced in the cities, we treat it as a random variable with different possible realization (or scenarios) - this leads to a more complicated two-stage integer stochastic optimization problem (see [2]), where the first-stage (or planning) decision is on the construction of the incinerator plants (before we get to "know" the particular realization of the random variable) and the second-stage decisions are the operating ones (transportation) that are carried out once we know the values of the produced waste. The notation we use to describe the model is summarized in the following table: 
Table 1: The notation

Indices and sets
$i \in N$
$k \in K$
$e \in A$
$s \in S$
$i c \in N_{c} \subset N$
$i t \in N_{t} \subset N$
$i s \in N_{s} \subset N$
$i l \in N_{l} \subset N$
Variables
$z$
$y_{e, s}$
$p_{i c, s}$
$x_{i s}$
$k_{i s, s}$

index of nodes
index of coordinates
index of arcs
index of scenarios
index of cities
index of traffic points
index of incinerators
index of landfills
total profit
amount of transported units
number of penalty units
decision to build the incinerator (binary)
capacity of the incinerator

\begin{tabular}{ll}
\multicolumn{2}{l}{ Parameters } \\
$n_{i, k}$ & coordinates of nodes \\
$M_{i, e}$ & incidence matrix \\
$c_{e}$ & transportation costs \\
$g_{s}$ & profit in the incinerator \\
$q_{s}$ & penalty for waste not removed \\
$t_{i c, s}$ & quantity of trash in cities \\
$d_{e}$ & distance by arcs \\
$P r_{s}$ & probability of scenario \\
$h_{s}$ & profit in landfills \\
$f$ & freight in CZK per km using lorry \\
$b$ & costs to build the incinerator \\
$u$ & upper bound for penalty
\end{tabular}

The transportation cost is expressed as $c_{e}=f \cdot d_{e}$. The production of waste in each city $t_{i c, s}$ differs according to the scenario $s$. In our case, we consider 3 scenarios, each with probability $\operatorname{Pr}_{s}=1 / 3, \forall s \in S$.

$$
t_{i c, s}= \begin{cases}+0 \%, & s=s_{1} \\ -20 \%, & s=s_{2} \\ +20 \%, & s=s_{3}\end{cases}
$$

The problem at hand can be written as:

$$
\begin{array}{rrr}
\max & -\sum_{i s} x_{i s} b+\sum_{s} \operatorname{Pr}_{s}\left(\sum_{i s} \sum_{e}-M_{i s, e} y_{e, s} g_{s}-\sum_{e} c_{e} y_{e, s}-\sum_{i c} p_{i c, s} q_{s}+\sum_{i l} \sum_{e}-M_{i l, e} y_{e, s} h_{s}\right) \\
\text { s.t. } & \sum_{i s} x_{i s} & \geq 1, \\
\sum_{e} M_{i c, e} y_{e, s} & \leq t_{i c, s}, & \forall i c, s, \\
\sum_{i c} p_{i c, s} & \leq u \sum_{i c} t_{i c, s}, & \forall s, \\
t_{i c, s}-\sum_{e} y_{e, s} M_{i c, e} & =p_{i c, s}, & \forall i c, s, \\
\sum_{e} M_{i t, e} y_{e, s} & =0, & \forall i t, s, \\
\left(\sum_{i c} t_{i c, s} x_{i s}\right. & =k_{i s, s}, & \forall i s, s, \\
-\left(\sum_{e} M_{i l, e} y_{e, s}\right) & \leq k_{i s, s}, & \forall i s, s, \\
x_{i s} & \in\{0,1\}, & \forall i s, \\
y_{e, s} & \geq 0, & \forall e, s, \\
p_{i c, s} & \geq 0, & \forall i c, s, \\
k_{i l, s} & \geq 0, & \forall i l, s .
\end{array}
$$

The different terms in the objective refer to the following: the first term $-\sum_{i s} x_{i s} b$ contains the first stage decision (costs for building incinerators), the next terms represent the second stage decisions - yields in landfills and incinerators $\sum_{i s} \sum_{e}-M_{i s, e} y_{e, s} g_{s}+\sum_{i l} \sum_{e}-M_{i l, e} y_{e, s} h_{s}$, transportation costs $-\sum_{e} c_{e} y_{e, s}$ and the penalty term, if we don't collect the waste $-\sum_{i c} p_{i c, s} q_{s}$.

The first constraint $\sum_{i s} x_{i s} \geq 1$, which states that at least one incinerator will be build, belongs to the first stage decision, whereas the rest of the constraints belong to the second stage. The next constraint, $\sum_{e} M_{i c, e} y_{e, s} \leq t_{i c, s}, \forall i c, s$, states that the transported units of waste cannot exceed the production in each city and each scenario. $\sum_{i c} p_{i c, s} \leq u \sum_{i c} t_{i c, s}, \forall s$ gives us the possibility not to collect all the waste, but we can pay the penalty instead up to $u \%$ of the total waste in the each scenario. The penalty is computed as the difference between the waste production and the number of transported units, $p_{i c, s}=t_{i c, s}-\sum_{e} y_{e, s} M_{i c, e}, \forall i c, s$. In our road network, we consider also traffic points. Traffic points can be interpreted as the main junctions and are mathematically represented as the nodes, in which the inflow equals the outflow. In the model (1), such nodes are underlined by $\sum_{e} M_{i t, e} y_{e, s}=0, \forall i t, s$ setting that there is no production of waste. The dependent variable $k_{i s, s}$ is computed as $k_{i s, s}=\left(\sum_{i c} t_{i c, s}\right) x_{i s}, \forall i s, s$ and is used in the last constraint, $-\left(\sum_{e} M_{i l, e} y_{e, s}\right) \leq k_{i s, s}, \forall i s, s$, where the inflow to incinerators cannot exceed the capacity of incinerators. 


\section{Data for the Real-World Problem}

In this section we provide an insight into the data for the real-world model (1). We have at our disposal historical data about the collection of the municipal waste during the years 2009 and 2015. Each city, landfill and incinerator has GPS coordinates. Potential coordinates of incinerators (denoted as WtE - waste to energy plants), and the regions they are located in, are listed in the table below.

Table 2: The positions of the possible incinerator plants

\begin{tabular}{llll} 
Node & Longitude & Latitude & Region \\
\hline $1 \mathrm{WtE}$ & 14.09 & 56.82 & Středočeský \\
$2 \mathrm{WtE}$ & 17.60 & 56.22 & Olomoucký \\
$3 \mathrm{WtE}$ & 15.77 & 56.84 & Pardubický \\
$4 \mathrm{WtE}$ & 14.37 & 56.86 & Hlavní město Praha \\
$5 \mathrm{WtE}$ & 16.60 & 55.92 & Jihomoravský \\
$6 \mathrm{WtE}$ & 14.37 & 56.86 & Hlavní město Praha \\
$7 \mathrm{WtE}$ & 15.13 & 56.57 & Středočeský \\
$8 \mathrm{WtE}$ & 14.81 & 57.02 & Středočeský \\
$9 \mathrm{WtE}$ & 14.37 & 56.86 & Hlavní město Praha \\
$10 \mathrm{WtE}$ & 17.75 & 56.51 & Moravskoslezský
\end{tabular}

To give an inside into how big a problem we are dealing with (in comparison with the example in the previous section), we list here the size of the different sets. We are considering municipalities with extended powers $i c \in N_{c},\left|N_{c}\right|=206$, already built landfills an incinerators $i l \in N_{l},\left|N_{l}\right|=114$, set of potential incinerators $i s \in N_{s},\left|N_{s}\right|=10$. The set of traffic points $i t \in N_{t}, N_{t}=\emptyset$ is an empty set, because the set of edges $e \in A$, $|A|=2069$, has sufficient cardinality for our purpose. This leads to the simplification of the model (1), in which the constraint $\sum_{e} M_{i t, e} y_{e, s}=0$ is redundant $\forall i t, s$. The rest of the model (1) holds.

The first stage decision $x_{i}$ is a binary variable and it denotes whether the incinerator is built or not. The second stage decision $y_{e, s}$ is made after the period of seven years and it denotes the number of transported waste. We are trying to model if the investment to build an incinerator will come back after the seven years or how much of the initial building costs will be paid back.

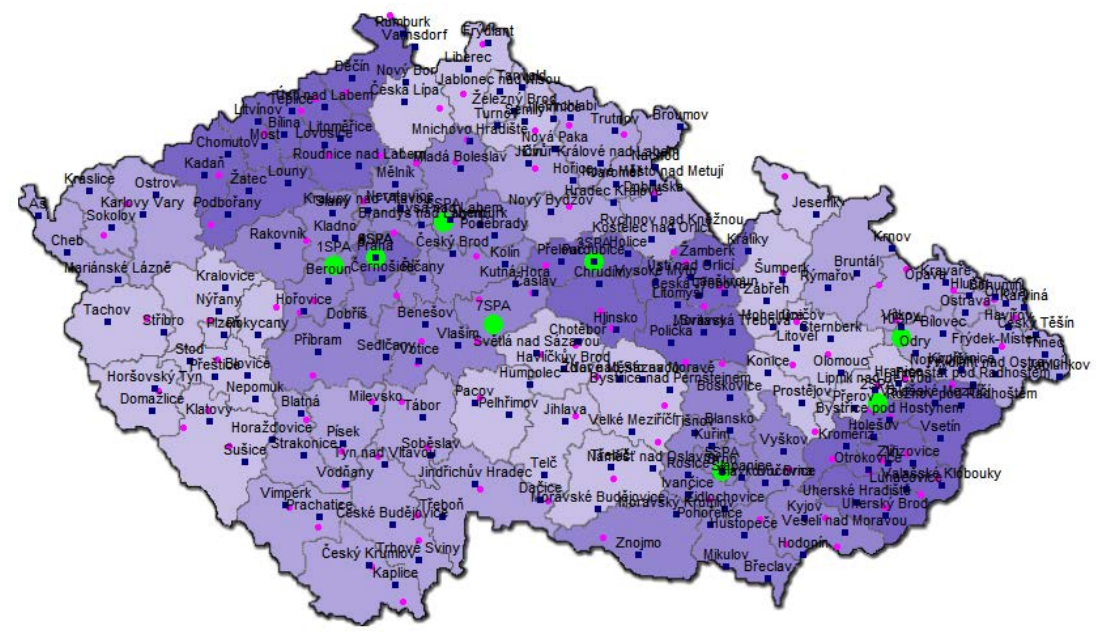

Figure 1: Layout of cities, landfills and possible incinerators.

The layout of cities (blue squares), landfills (magenta circles) and possible incinerators (green circles) can be seen in the Figure 1. Regions are represented by different shade of violet. The waste distribution among regions in percentage is shown in the Figure 2.

\section{PHA and Results}

Stochastic programs (SP) arise in a variety of situations. Examples can be found in server location, electricity generation, supply chain design, network and so on. The structure of such programs makes them, in general, difficult to solve. We can however obtain an easier solution using a special structure.

A common approach how to represent uncertainty is to formulate a finite number of discrete scenarios associated with probabilities for the values of uncertain parameters. Decisions depend on the number of stages according to which parameter values are assumed to be known to the decision-maker and when the decisions 
must be made. If we combine the constraints of our problem with an objective to minimize expected cost (by "cost" we can mean also some measure of risk), the resulting SP can become very large. The progressive hedging algorithm (PHA) represents an effective method for solving multistage SP.

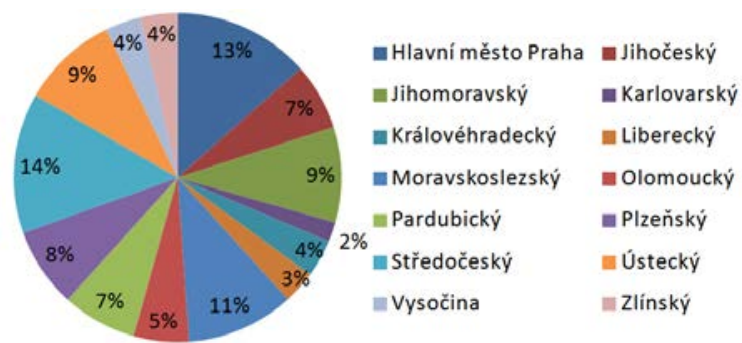

Figure 2: Waste distribution in regions.

\section{The Progressive Hedging Algorithm for the two-stage stochastic mixed-integer problem}

To avoid a "collision of notation" with the presented models, we describe the algorithm with the following notation: $\lambda(s)$ will denote the first-stage decision (depending on the particular scenario $s$ ), $\mu(s)$ the secondstage decision, $\alpha$ the first-stage cost vector, $\beta(s)$ the second-stage cost vector. Furthermore, to ease the notation, we collapse all the constraints (or, more precisely, the set that the constraints define) for a particular scenario (in both stages) into $\Gamma(s)$.

Step 1: Initialization: iteration $\iota=0$, price term $w^{\iota}(s)=0, \forall s \in S$. For each $s \in S$ compute the individual scenario solution $x(s)$ (with components $\lambda(s)$ and $\mu(s))$ :

$$
\begin{array}{cc}
\max & \alpha^{T} \lambda(s)+\beta(s)^{T} \mu(s) \\
\text { s.t. } & \lambda(s), \mu(s) \in \Gamma(s) .
\end{array}
$$

Step 2: Iteration update: $\iota=\iota+1$.

Step 3: Aggregation: $\hat{\lambda}^{\iota}=\sum_{s \in S} \operatorname{Pr}_{s} \lambda^{\iota}(s)$.

Step 4: Price update: $w^{\iota}(s)=w^{\iota-1}(s)+r\left(\lambda^{\iota}(s)-\hat{\lambda}^{\iota}(s)\right)$.

Step 5: Decomposition: $\forall s \in S$ compute:

$$
\begin{array}{cc}
\max & \alpha^{T} \lambda(s)+\beta(s)^{T} y(s)-w^{\iota}(s)^{T} \lambda(s)-\frac{r}{2}\left\|\lambda(s)-\hat{\lambda}^{\iota}\right\|^{2} \\
\text { s.t. } & \lambda(s), \mu(s) \in \Gamma(s) .
\end{array}
$$

Step 6: Termination: If all scenario solutions $\lambda(s)$ are equal, stop. Else, go to Step 2.

In Step 1 of the algorithm, we solve the model (1) for each scenarios separately. In Step 5, the model (1) is transformed into:

$$
\begin{array}{rrr}
\max -\sum_{i s} x_{i s} b+\sum_{i s} \sum_{e}-M_{i s, e} y_{e, s} g_{s}-\sum_{e} c_{e} y_{e, s}-\sum_{i c} p_{i c, s} q_{s}+ & \\
& +\sum_{i l} \sum_{e}-M_{i l, e} y_{e, s} h_{s}+\sum_{i s}-\left(x_{i s} w_{i s, s}-\frac{r}{2}\left\|x_{i s}-\hat{x}_{i s}\right\|^{2}\right) & \\
\sum_{i s} x_{i s} & \geq 1, & \\
\text { s.t. } & \sum_{e} M_{i c, e} y_{e, s} & \leq t_{i c, s}, \\
\sum_{i c} p_{i c, s} & \leq u \sum_{i c} t_{i c, s}, & \forall i c, s, \\
t_{i c, s}-\sum_{e} y_{e, s} M_{i c, e} & =p_{i c, s}, & \forall s, \\
\left(\sum_{i c} t_{i c, s}\right) x_{i s} & =k_{i s, s}, & \forall i c, s, \\
-\left(\sum_{e} M_{i l, e} y_{e, s}\right) & \leq k_{i s, s}, & \forall i s, s, \\
x_{i s} & \in\{0,1\}, & \forall i s, \\
y_{e, s} & \geq 0, & \forall e, s, \\
p_{i c, s} & \geq 0, & \forall i c, s, \\
k_{i l, s} & \geq 0, & \forall i l, s,
\end{array}
$$

where $r>0$ is a PHA parameter and $w_{i s, s}$ is a price vector (whose computation is described in Step 4 of the PHA algorithm). Athough the model (4) is not a convex optimization problem (since we have binary variables), 
its continuous relaxation is convex - the objective is a linear function plus a sum of squares and the constraints are all linear. For further information see [4].

The following values of parameters were used in the numerical study $f=3, b=500,000,000, h \in(50 ; 80)$, $q \in(10,000 ; 15,000)$ and $r=10^{8}$. These value were chosen after careful consideration and a thorough discussion with experts on waste management. Furthermore, we conducted an analysis of the impact of the incinerator profit parameter $g$ on the solution. The results are best summarized in Fig. 3 and Fig. 4. When the profit of the incinerator plants is low (Fig. 3), there seems to be just a small incentive to build these plants and most of the waste is instead "processed" in landfills - the optimal objective value of this problem setting was 455.4 million CZK. However, as the incinerator profit rises, the model encourages us to build more incinerator plants with the majority of waste processed in these plants - the optimal objective value of this problem setting increased to 3,121.4 million CZK.

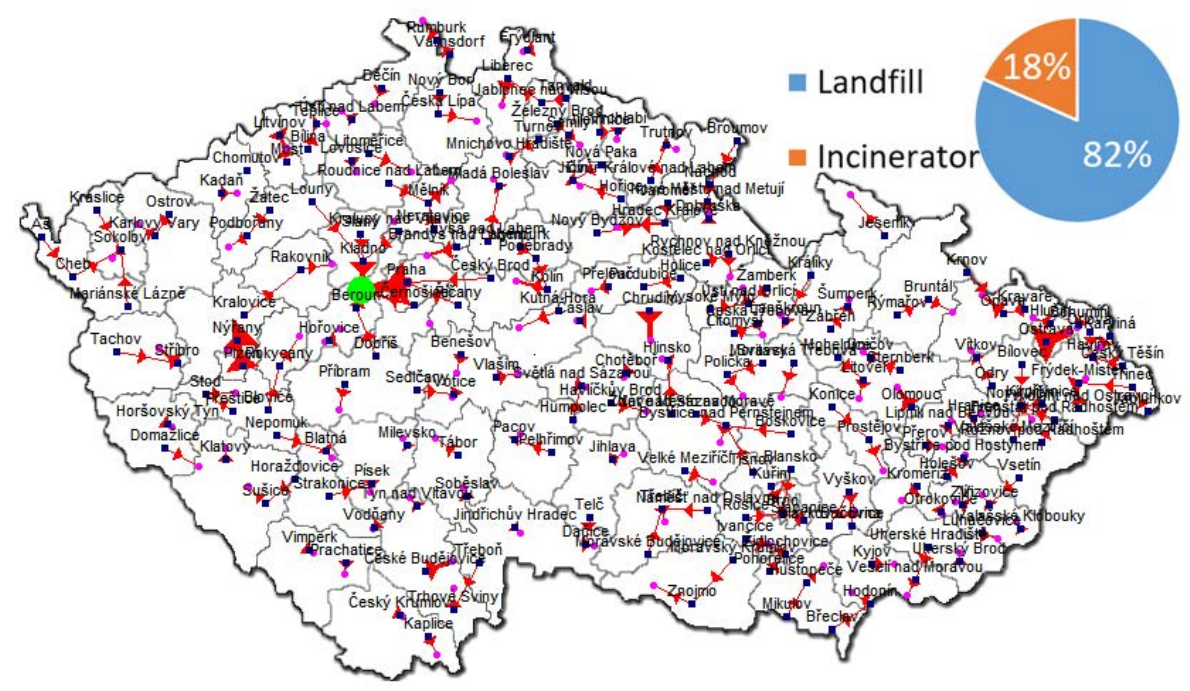

Figure 3: Selected incinerators (green), transportation flows (red) and waste treatment (chart) for $g \in(100 ; 150)$.

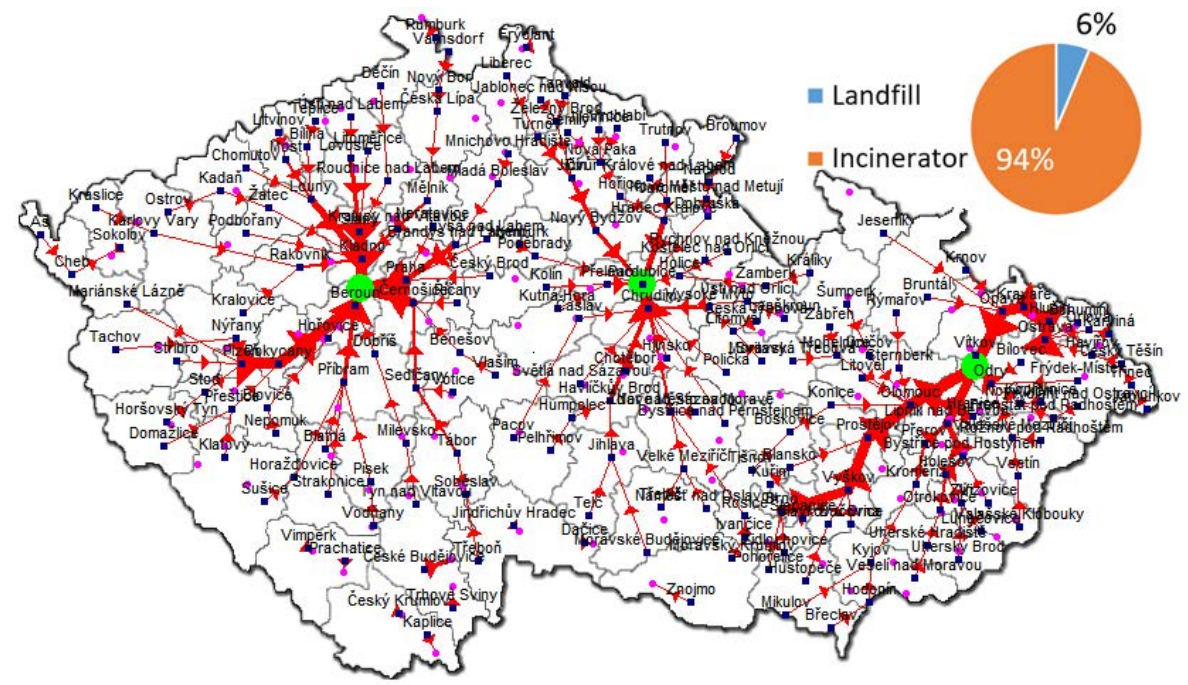

Figure 4: Selected incinerators (green), transportation flows (red) and waste treatment (chart) for $g \in(400 ; 500)$.

\section{Conclusion}

The paper presents and discusses results of the original implementation of the Progressive Hedging Algorithm (PHA) for the specific scenario-based two-stage stochastic mixed-integer linear program that has been designed for the studied real-world waste management problem. At first, an overview of related waste management problems is given and the modeling challenge is stated as the task to find suitable places for new incinerators, to optimize their capacities and related waste transport. The model building phase utilizes the notation rules inherited from the application area for the model elements (indices, parameters, variables). The proposed model 
follows two-stage decision structure and its solved instance is based on the data for the Czech Republic. Because of the real-world problem's size PHA is applied as a solution technique and obtained results are visualized by figures involving coloured maps. The use of PHA algorithm is also suitable for the future research on more advanced models including pricing and advertising nonlinear terms (see [7]). Unfortunately, the convexity of the relaxed model will not be achieved, so the PHA will serve as the part of the heuristic solution technique proposed (such as the one in [13]) in the future.

Acknowledgement: This paper has been supported by the project "Computer Simulations for Effective Low-Emission Energy" funded as project No. CZ.02.1.01/0.0/0.0/16_026/0008392 by Operational Programme Research, Development and Education, Priority axis 1: Strengthening capacity for high-quality research. The authors gratefully acknowledge financial support provided by ERDF within the research project No. CZ.02.1.01/0.0/0.0/16_026/0008413 "Strategic Partnership for Environmental Technologies and Energy Production". This work was also supported by The Ministry of Education, Youth and Sports of the Czech Republic, INTER-COST project LTC18053 and European COST Action CA15140 and by IGA BUT: No. FSI-S-17-4785.

\section{References}

[1] Bazaraa, M.S., Jarvis, J.J., and Sherali, H.D.: Linear programming and network flows. John Wiley and Sons, New York (1990)

[2] Birge, J.R., Louveaux, F.: Introduction to Stochastic Programming. Springer, New York (1997)

[3] Dolezel, P., Heckenbergerova, J., Mariska, M., and Skrabanek, P.: Transportation schedule-respected production planning using genetic algorithm based approach. Mendel 21(1), 31-38 (2015)

[4] Gade, D., Hackebeil, G., Ryan, S.M., Watson, J.-P., Wets, R.J.-B., and Woodruff, D.L.: Obtaining lower bounds from the progressive hedging algorithm for stochastic mixed-integer programs. Mathematical Programming 157(1), 47-67 (2016)

[5] Hrabec, D., Popela, P., Roupec, J., Jindra, P., and Novotný, J.: Hybrid algorithm for wait-and-see transportation network design problem with linear pricing: Mendel 21(1), 183-188 (2015)

[6] Hrabec, D., Viktorin, A., Somplak, R., Pluhacek, M., and Popela, P.: A heuristic approach to the facility location problem for waste management: A case study. Mendel 22(1), 61-66 (2016)

[7] Hrabec, D., Šomplák, R., Nevrlý, V., and Smejkalová, V.: Sustainable model integration of waste production and treatment process based on assessment of GHG. Chemical Engineering Transactions 70(1), $1603-1608$ (2018)

[8] Janostak, F., Pavlas, M., Putna, O., Somplak, R., and Popela, P.: Heuristic approximation and optimization for waste-to-energy capacity expansion problem. Mendel 22(1), 123-130 (2016)

[9] Kudela J., Popela P.: Two-stage stochastic facility location problem: GA with benders decomposition. Mendel 21(1), 53-58 (2015)

[10] Kudela, J., Popela, P., Somplak, R., Malek, M., Rychtar, A., and Hrabec, D.: The L-shaped method for large-scale mixed-integer waste management decision making problems. Chemical Engineering Transactions 61(1), 1087-1092 (2017)

[11] Kudela, J., Popela, P.: Warm-start cuts for generalized benders decomposition. Kybernetika 53(6), 10121025 (2017)

[12] Kudela, J., Somplak, R., Nevrly, V., and Lipovsky, T.: Robust waste transfer station planning by stochastic programming. Chemical Engineering Transactions 70(1), 889-894 (2018)

[13] Marada, T., Matousek, R., and Zuth, D.: Design of linear quadratic regulator (LQR) based on genetic algorithm for inverted pendulum. Mendel 23(1), 149-156 (2017)

[14] Nevrly, V., Somplak, R., Popela, P., Pavlas, M., Osicka, O., and Kudela, J.: Heuristic challenges for spatially distributed waste production identification problems. Mendel 22(1), 109-116 (2016)

[15] Osicka, O., Hrdina, J., Somplak, R., Popela, P., and Pavlas, M.: Shapley value approximation for games with distant players. Mendel 22(1), 103-108 (2016)

[16] Pavlas, M., Nevrly, V., Popela, P., and Somplak R.: Heuristic for generation of waste transportation test networks. Mendel 21(1), 189-194 (2015)

[17] Pavlas, M., Somplak, R., Smejkalova, V., Nevrly, V., Zaviralova, L., Kudela, J., and Popela, P.: Spatially distributed production data for supply chain models - Forecasting with hazardous waste. Journal of Cleaner Production 161(10), 1317-1328 (2017)

[18] Rardin, R.L.: Optimization in Operations Research. Second edition, Person, Hoboken, New Jersey (2015)

[19] Smejkalova, V., Somplak, R., Nevrly, V., and Pavlas, M.: Heuristic methodology for forecasting of quantities in waste management. Mendel 23(1), 185-192 (2017) 